\title{
Medium-Resolution s-process Element Survey of 47 Tuc Giant Stars
}

\author{
C. C. Worley $^{\mathrm{A}, \mathrm{D}}$ and P. L. Cottrell ${ }^{\mathrm{B}, \mathrm{C}}$

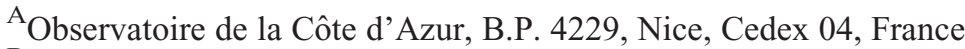 \\ ${ }^{\mathrm{B}}$ The Beatrice Tinsley Institute, Department of Physics \& Astronomy, University of Canterbury, \\ Private Bag 4800, Christchurch, New Zealand \\ ${ }^{C}$ Max Planck Institut für Astrophysik, Karl-Schwarzschild Str 1, 85741, Garching, Germany \\ ${ }^{\mathrm{D}}$ Corresponding author. Email: cworley@oca.eu
}

\begin{abstract}
Medium-resolution $(R \sim 6500)$ spectra of 97 giant stars in the globular cluster 47 Tucanæ (47 Tuc) have been used to derive the $\mathrm{C}$ - and $\mathrm{N}$-abundance sensitive index, $\delta \mathrm{C}$, and to infer abundances of several key elements (Fe, $\mathrm{Na}, \mathrm{Si}, \mathrm{Ca}, \mathrm{Zr}$ and $\mathrm{Ba}$ ) for a sample of 13 of these stars with similar effective temperature $\left(T_{\text {eff }}\right)$ and surface gravity $(\log g)$. These stars have stellar properties similar to the well-studied $47 \mathrm{Tuc}$ giant star, Lee 2525 , but with a range of $\mathrm{CN}$ excess $(\delta \mathrm{C})$ values which are a measure of the $\mathrm{CN}$ abundance. The $\delta \mathrm{C}$ index is shown to be correlated with $\mathrm{Na}$ abundance for this sample, confirming previous studies. $\mathrm{Fe}, \mathrm{Ca}, \mathrm{Si}$ and the light and heavy $s$-process (slow neutron capture) elements, $\mathrm{Zr}$ and $\mathrm{Ba}$, respectively, have a narrow range of abundance values, indicative of a homogeneous abundance within this population of stars. The constancy of many element abundances ( $\mathrm{Fe}, \mathrm{Si}, \mathrm{Ca}, \mathrm{Zr}, \mathrm{Ba}$ ) and the $\delta \mathrm{C}$ and $\mathrm{Na}$ abundance correlation could imply that there has been a second era of star formation in this cluster that has revealed the products of CNO-cycle burning via hot bottom burning (depletion of $\mathrm{C}$, enhancement of $\mathrm{N}$ and the production of $\mathrm{Na}$ for high $\delta \mathrm{C}$ population). But there is no overall metallicity change across the range of $\delta \mathrm{C}$ values at a given position in the HR diagram as has been seen in some other globular clusters.
\end{abstract}

Keywords: globular clusters: individual (47 Tuc) — stars: abundances — stars: late-type

Received 2011 September 13, accepted 2011 October 14, published online 2011 December 16

\section{Introduction}

Globular clusters (GC) are relics of the birth of the Milky Way galaxy. Their ages, on the order of the age of the Universe, mean that they hold information about early stellar and galactic formation and evolution processes. In particular, as closed systems, their chemical evolution can be explored through the chemical signatures of their current stellar components. These signatures are a combination of current nuclear processes within the observed stars and of the material from which the stars formed, and hence of the previous generations of stars that polluted the intra-cluster medium. These signatures are comprised of both light and heavy element abundances, and it is by disentangling these signatures that the pollution and evolution events of GCs can be revealed.

A key goal of our survey of 47 Tuc giant stars was to ascertain the feasibility of determining $s$-process element abundances from spectra obtained at the optimal resolution of the AAOmega spectrograph at the AngloAustralian Telescope (AAT; $R \sim 8000$ ). These $s$-process elements are produced via the slow (compared to $\beta$-decay rate) accumulation of neutrons onto iron seed nuclei that can produce all the elements up to lead. Previous highresolution studies of $s$-process elements in 47 Tuc were limited to samples of less than 10 stars. Worley et al. (2010) and Wylie et al. (2006) observed homogeneities and variations in the $s$-process abundance distributions, respectively. A statistically significant sample is necessary in order to truly characterise the $s$-process abundance distribution of 47 Tuc. This survey was designed to test the resolution limits of abundance determination for weak $s$-process features in giant stars.

Our survey was also designed to obtain light-element abundances alongside the $s$-process elements for a more complete chemical analysis of these stars. There are several key light-elemental abundance anomalies that have been observed in globular cluster stars, in particular relating to variations in carbon, nitrogen, oxygen, sodium, magnesium and aluminium. Initially, from low-resolution spectra, indices on the $\mathrm{CN}$ and $\mathrm{CH}$ molecular bands determined an anti-correlation between $\mathrm{CH}$ and $\mathrm{CN}$ in globular cluster stars (Norris \& Freeman 1979), whereby $\mathrm{CN}$-weak stars are defined generally as having solar $\mathrm{C}$ and $\mathrm{N}$ abundances, while for $\mathrm{CN}$-strong stars $\mathrm{N}$ is enhanced by up to +1 dex and $\mathrm{C}$ is depleted by $\sim-0.4$ dex (Cannon et al. 2003).

Further study on the $\mathrm{CN}$ bimodality showed that while some clusters showed evidence of both $\mathrm{CN}$-weak and $\mathrm{CN}$-strong stars at all stages of evolution (e.g. 47 Tuc, Cannon et al. 1998), others had only one or the other at different stages, although typically both were present on the red giant branch (RGB; Gratton et al. 2004, and 
references therein). The bimodality was noted to be fairly consistent from the main sequence (MS) to the RGB for any particular globular cluster, but the main discrepancy came in the asymptotic giant branch (AGB) stars where the number of $\mathrm{CN}$-strong stars decreased significantly or there were none at all (Campbell et al. 2006; Sneden et al. 2000). It was noted that this could simply be due to a lack of observations of AGB stars, and that increasing the AGB sample to compare to those from the RGB may bear useful results. Campbell et al. (2010) presented preliminary results on such an observational programme which significantly increased the sample sizes of AGB stars for 10 GCs. The preliminary results confirmed that AGB stars in GCs tended to be, in the majority, $\mathrm{CN}$-weak.

Variations in $\mathrm{O}, \mathrm{Na}, \mathrm{Al}$ and $\mathrm{Mg}$ have also been observed and appear to be related to the variations in $\mathrm{C}$ and N (Cottrell \& Da Costa 1981). H-burning via the $\mathrm{CNO}, \mathrm{NeNa}$ and $\mathrm{MgAl}$ cycles affects the abundances of all of these elements. The variations that have been observed are consistent with $\mathrm{CNO}$ cycle processing, where $\mathrm{Na}, \mathrm{Al}$ and $\mathrm{Mg}$ are correlated to $\mathrm{CN}$ strength, while $\mathrm{O}$ is anti-correlated. However, the observed variations also seem to be correlated with the cluster metallicity. The correlation of $\mathrm{Na}, \mathrm{Al}$ and $\mathrm{Mg}$ with $\mathrm{CN}$ strength is seen in metal-poor clusters, that of $\mathrm{Na}$ and $\mathrm{Al}$ with $\mathrm{CN}$ strength in less metal-poor clusters and only $\mathrm{Na}$ is correlated with $\mathrm{CN}$ strength in metal-rich clusters (Gratton et al. 2004).

These variations in light elements can be attributed to H-burning via the CNO cycle during hot bottom burning in intermediate-mass AGB stars. Sufficiently high temperatures exist at the bottom of the envelope (top of the H-burning shell) in these stars to allow these events to occur (Cottrell \& Da Costa 1981). The CN bimodality in particular is thought to be evidence of a previous generation of $\mathrm{CN}$-weak stars polluting the star forming material such that the $\mathrm{CN}$-strong population are born with material already fully processed in the CNO cycle. The thermally pulsing stage of AGB evolution is also responsible for producing enhancements in light and heavy $s$-process elements (Karakas \& Lugaro 2010).

\section{Observations}

In October 2008, 97 giant stars in 47 Tuc were observed using AAOmega on the AAT (Sharp et al. 2006). The target stars were selected from: Lee (1977); Paltoglou \& Freeman (1984); Brown \& Wallerstein (1992); Wylie et al. (2006); and Worley et al. (2008). Photometry was provided by G. Da Costa and F. Grundahl (private communication). The coordinates of the sample were verified using 2MAss (Skrutskie et al. 2006) where $J$ and $K$ magnitudes were also obtained. ${ }^{1}$

Figure 1 shows the location of the 47 Tuc survey stars in the $V-(B-V)$ colour-magnitude diagram (CMD). The mask configurations for AAOmega restricted stars to within a 2-magnitude range. In order to obtain stars on the

\footnotetext{
${ }^{1}$ http: / / www. ipac.caltech.edu/2mass
}

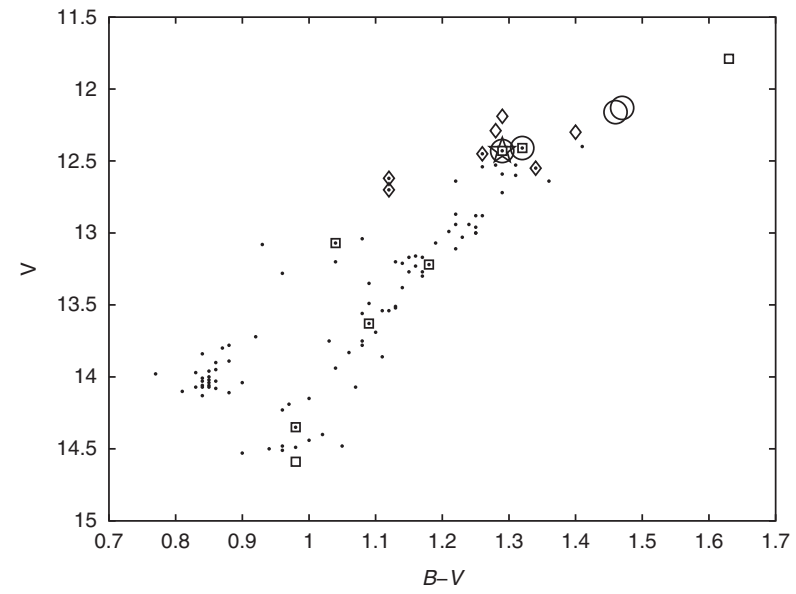

Figure 1 Colour-magnitude diagram of the 47 Tuc stars observed in the AAOmega medium-resolution survey $(\bullet)$. Stars from Brown \& Wallerstein (1992) $(\bigcirc)$, Wylie et al. (2006) $(\diamond)$ and Worley et al. (2008) ( $\square$ ), as well as Lee 2525 (star) are shown.

Table 1. Wavelength regions and key spectral features observed in the AAOmega 47 Tuc survey

\begin{tabular}{ll}
\hline $\begin{array}{l}\text { Camera:grating } \\
\text { regions }\end{array}$ & Features $(\AA)$ \\
\hline $\begin{array}{l}\text { Blue:3200B } \\
\lambda \lambda 4040-4350 \AA\end{array}$ & CN $(4142-4216)$ \\
& CH (4300-4330 G Band) \\
Red:2000R & \\
$\lambda \lambda 5750-6200 \AA$ & Na I $(6154.23,6160.75)$ \\
& Si I $(6131.57,6131.85,6145.02,6155.13)$ \\
& Ca I $(6156.02,6166.44,6169.56)$ \\
& Fe I $(6147.83,6157.73)$ \\
& Zr I $(6143.18)$ \\
& Ba II $(6141.73)$ \\
& La II $(5805.77)$ \\
& Nd II $(5811.57)$ \\
&
\end{tabular}

tip of the AGB and on the RGB below the horizontal branch (HB), the range of magnitudes for this survey was $12.5 \leq V \leq 14.5$. Hence there were some stars from Brown \& Wallerstein (1992) and Wylie et al. (2006) that could not be observed as they were outside this magnitude range.

AAOmega has a red and a blue camera with which stars can be observed simultaneously over two distinct wavelength ranges. The wavelength regions observed for this survey and the key features that have been analysed are listed in Table 1. The resolution of the spectra was $R \sim 6500$ and the signal-to-noise (SNR) per pixel ranged from 30 to 50 for the blue arm, and 60 to 90 for the red arm. The spectra were reduced using the reduction pipeline $2 \mathrm{DFDR}^{2}{ }^{2}$

The normalisation of the spectra was treated differently for the blue and red arms. The key features in the blue-arm observations were $\mathrm{CN}$ and $\mathrm{CH}$ molecular bands that obscured the location of the true continuum. In the red-arm observations, molecular features were less

\footnotetext{
${ }^{2}$ http: / / www . aao.gov.au/2df/manual/UsersManual.pdf
} 
Table 2. Elemental abundances and abundance ratios with respect to the Sun derived from high-resolution (HR) and medium-resolution (MR) spectra of Arcturus

\begin{tabular}{|c|c|c|c|c|}
\hline$\overline{\text { Species }}$ & $\lambda(\AA)$ & $\chi(\mathrm{eV})$ & $\mathrm{HR}$ & MR \\
\hline$[\mathrm{Fe} \mathrm{I} / \mathrm{H}]$ & 6136.62 & 2.45 & -0.63 & -0.44 \\
\hline$[\mathrm{Fe} \mathrm{I} / \mathrm{H}]$ & 6147.83 & 4.08 & -0.61 & -0.62 \\
\hline \multirow[t]{3}{*}[\mathrm{Fe}\mathrm{I}/\mathrm{H}]{} & 6157.73 & 4.08 & -0.60 & -0.52 \\
\hline & & $\langle[\mathrm{Fe} / \mathrm{H}]\rangle$ & -0.61 & -0.53 \\
\hline & & $\sigma$ & 0.02 & 0.09 \\
\hline$[\mathrm{Na} \mathrm{I} / \mathrm{Fe}]$ & 6154.23 & 2.10 & 0.13 & 0.02 \\
\hline \multirow[t]{3}{*}[\mathrm{Na}\mathrm{I}/\mathrm{Fe}]{} & 6160.75 & 2.10 & 0.19 & 0.10 \\
\hline & & $\langle[\mathrm{Na} / \mathrm{Fe}]\rangle$ & 0.16 & 0.06 \\
\hline & & $\sigma$ & 0.04 & 0.06 \\
\hline$[\mathrm{Si} \mathrm{I} / \mathrm{Fe}]$ & 6131.57 & 5.62 & 0.20 & 0.21 \\
\hline [Si I/Fe] & 6131.85 & 5.62 & 0.26 & 0.22 \\
\hline [Si I/Fe] & 6145.02 & 5.62 & 0.24 & 0.18 \\
\hline \multirow[t]{3}{*}[\mathrm{Si}/\mathrm{Fe}]{} & 6155.13 & 5.62 & 0.24 & 0.41 \\
\hline & & $\langle[\mathrm{Si} / \mathrm{Fe}]\rangle$ & 0.24 & 0.26 \\
\hline & & $\sigma$ & 0.03 & 0.10 \\
\hline$[\mathrm{Ca} \mathrm{I} / \mathrm{Fe}]$ & 6156.02 & 2.52 & 0.24 & 0.27 \\
\hline \multirow[t]{3}{*}[\mathrm{Ca}\mathrm{I}/\mathrm{Fe}]{} & 6166.44 & 2.52 & 0.36 & 0.23 \\
\hline & & $\langle[\mathrm{Ca} / \mathrm{Fe}]\rangle$ & 0.30 & 0.25 \\
\hline & & $\sigma$ & 0.08 & 0.03 \\
\hline$[\mathrm{Zr} \mathrm{I} / \mathrm{Fe}]$ & 6143.18 & 0.07 & 0.02 & -0.12 \\
\hline$[\mathrm{Ba} \mathrm{II} / \mathrm{Fe}]$ & 6141.73 & 0.70 & -0.20 & 0.04 \\
\hline$[\mathrm{La} \mathrm{II} / \mathrm{Fe}]$ & 5805.77 & 0.13 & -0.01 & -0.05 \\
\hline$[\mathrm{Nd} \mathrm{II} / \mathrm{Fe}]$ & 5811.57 & 0.86 & 0.00 & 0.00 \\
\hline
\end{tabular}

prominent at the stellar temperatures of these stars. In both cases comparison was made to the high-resolution spectrum of Arcturus (Hinkle \& Wallace 2005) which was convolved to a resolution comparable to the AAOmega observations. Different methods were employed in order to locate the continuum in the blue and the red.

For the red arm, the convolved Arcturus spectrum was compared with synthesised spectra generated using the Arcturus stellar model determined in Worley et al. (2009). Using the spectrum synthesis programme MOOG (Sneden 1973), the atomic linelist, collated from the latest laboratory values, was calibrated so that the synthetic spectrum matched the high-resolution spectrum for Arcturus. The linelist was modified to include hyperfine splitting components and isotopic ratios for barium. The abundances for each of the key lines in the red (see Table 1) were measured by spectrum synthesis for both the high- and medium-resolution spectra of Arcturus. Corrections for departures from local thermodynamic equilibrium for the two sodium features were applied after the abundance determination for each star (Lind et al. 2011). The abundances for Arcturus are listed in Table 2.

The $\mathrm{Ba}$ abundance shows the greatest change between the high-resolution and the convolved Arcutrus spectra. The $\mathrm{Ba}$ spectral line that was measured is very strong $\left(W_{\lambda} \approx 180 \mathrm{m \AA}\right)$ and so it is sensitive to changes in microturbulence. The remaining elements are in reasonable agreement to $\leq 0.15$ dex between the highresolution and convolved spectra. These variations in abundance from the high-resolution spectrum to the convolved medium-resolution spectrum provide a measure of the uncertainty in determining the abundances in medium-resolution spectra.

For the blue-arm spectral region the high-resolution Arcturus atlas was compared to the convolved Arcturus spectrum in the region of the $\mathrm{CN}$ and $\mathrm{CH}$ molecular bands. This allowed us to identify pseudo- continuum regions that could be used to normalise the spectra. By enforcing a ratio between the location of three wavelength regions (around $4090 \AA, 4220 \AA$ and $4318 \AA$ ), we created a linearly interpolated profile for each spectrum that was used to undertake the normalisation. For the spectra of 22 objects, there was no $4318 \AA$ region in the spectrum because of the instrument setup and placement of these objects in the focal plane of AAOmega. For these spectra, a normalisation based on the normalisation shape for stars of similar $T_{\text {eff }}$ was used.

\section{$3 \mathrm{CN}$ indices}

$\mathrm{CN}$ indices for stars in 47 Tuc have been measured in several studies. A number of stars observed in this survey were previously observed by Norris \& Freeman (1979) and Paltoglou \& Freeman (1984) (hereafter NF79 and PF84 respectively), in which they were classified by their $\mathrm{CN}$ indices, designated here as $\delta \mathrm{C}_{1979}$. This study seeks to extend these analyses. The $\mathrm{CN}$ index used here $\left(\delta \mathrm{C}_{2011}\right)$ was that defined by NF79 and PF84:

$$
S(4142)=-2.5 \log _{10}\left\{\frac{\int_{4120}^{4216} F_{\lambda} d \lambda}{\int_{4216}^{4290} F_{\lambda} d \lambda}\right\} .
$$

NF79 calibrated this line-intensity version of the CN index to the previous photometric index, C(4142), using the following equation:

$$
C(4142)=0.742 \times S(4142)+0.236 .
$$

In order to determine the $\mathrm{CN}$ excess, $\delta \mathrm{C}(4142)$ (hereafter $\delta \mathrm{C})$, the $\mathrm{CN}$ indices were considered in $\mathrm{C}(4142)-V$ space in NF79, and in $\mathrm{C}(4142)-(B-V)$ space in PF84. Based on these studies, in this analysis we used the following equation to derive $\delta \mathrm{C}_{2011}$ for the 47 Tuc sample:

$$
\delta C_{2011}=C(4142)-(0.304 \times(B-V)-0.275) .
$$

The stars were classified as $\mathrm{CN}$-weak if $\delta \mathrm{C}_{2011} \leq 0.11$ or $\mathrm{CN}$-strong if $\delta \mathrm{C}_{2011}>0.11$ (PF84), as shown in Figure 2 . The photometry, $T_{\text {eff }}$ and $\log g$ calculated from $V-K$, and $\delta \mathrm{C}_{2011}$ values for each star in the survey are given in Appendix 1. The majority of the stars fall along the $1: 1$ relation with a $2 \sigma$ uncertainty. There are three stars which lie outside the $2 \sigma$ limits.

In each case the star has changed designation between $\mathrm{CN}$-strong and $\mathrm{CN}$-weak. Lee $1506\left(\delta \mathrm{C}_{2011}=0.016\right)$ 


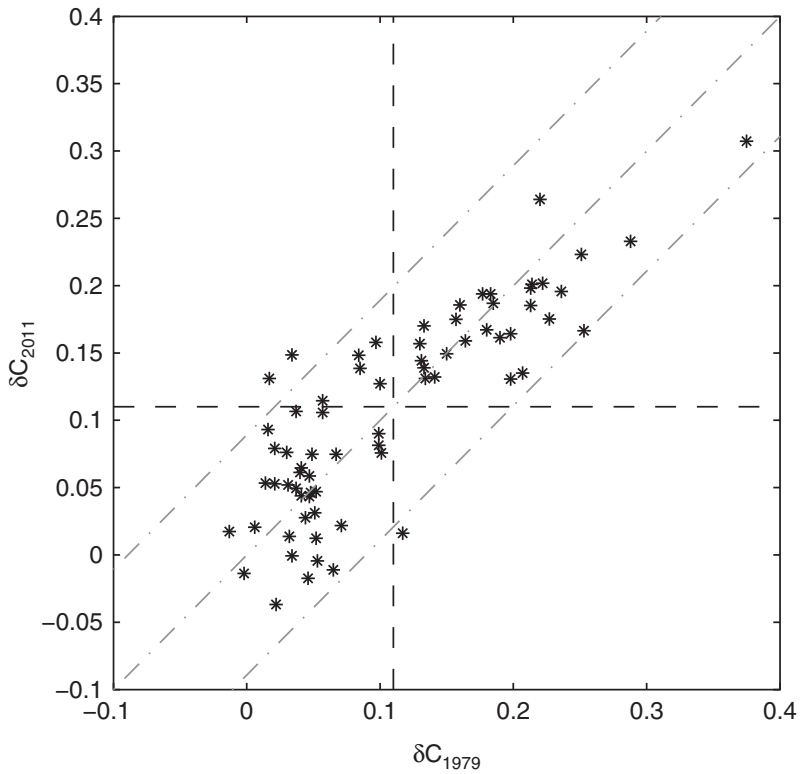

Figure 2 Comparison of $\mathrm{CN}$ excesses $(\delta \mathrm{C})$, from NF79 and PF84 (on abcissa) with the values derived in this study (on ordinate). The dashed lines indicate the $\mathrm{CN}$-weak/CN-strong threshold at $\delta \mathrm{C}=0.11$. The dash-dot lines indicate the $1: 1$ relation $\pm 2 \sigma$.

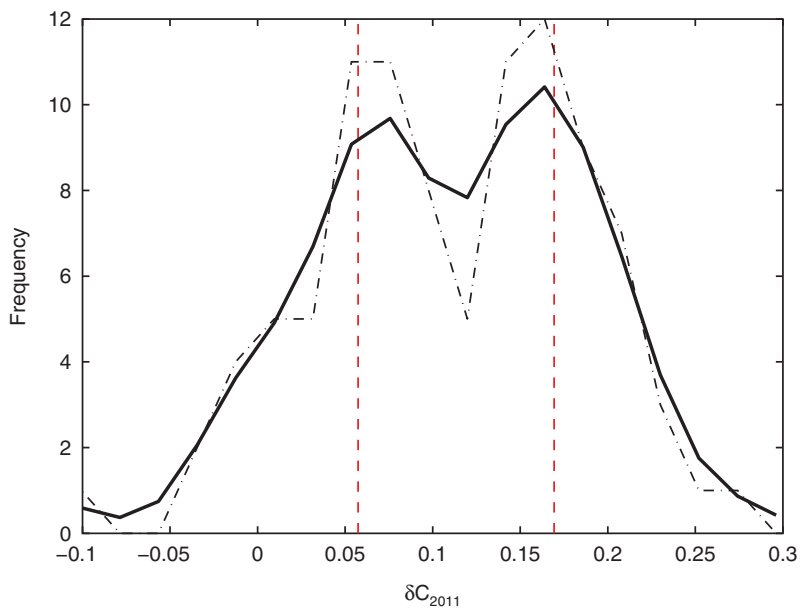

Figure 3 Frequency distribution, raw and smoothed, showing the $\mathrm{CN}$ bimodality in the 47 Tuc survey using the $\delta \mathrm{C}_{2011}$ values. The means of each population are shown as red lines.

changed from $\mathrm{CN}$-strong to $\mathrm{CN}$-weak, whereas Lee 5703 $\left(\delta \mathrm{C}_{2011}=0.131\right)$ and Lee $3415\left(\delta \mathrm{C}_{2011}=0.149\right)$ went from $\mathrm{CN}$-weak to $\mathrm{CN}$-strong. The spectra of these outliers were checked for any artifact or normalisation discrepancy but in all three cases the $\delta \mathrm{C}_{2011}$ value could not be reconciled with the previous measurements. This may indicate misidentification in the previous studies as the coordinates that are used here are consistent with the coordinates of these objects as given in SIMBAD. ${ }^{3}$

Figure 3 reduces the $\mathrm{CN}$ excess measurements of this study into a histogram using a bin interval of 0.02 (dotted line) and then smoothed using a Gaussian filter with a full width at half maximum (FWHM) of 0.05 (solid line) that

\footnotetext{
${ }^{3}$ http: / / simbad.u-strasbg.fr/simbad/
}
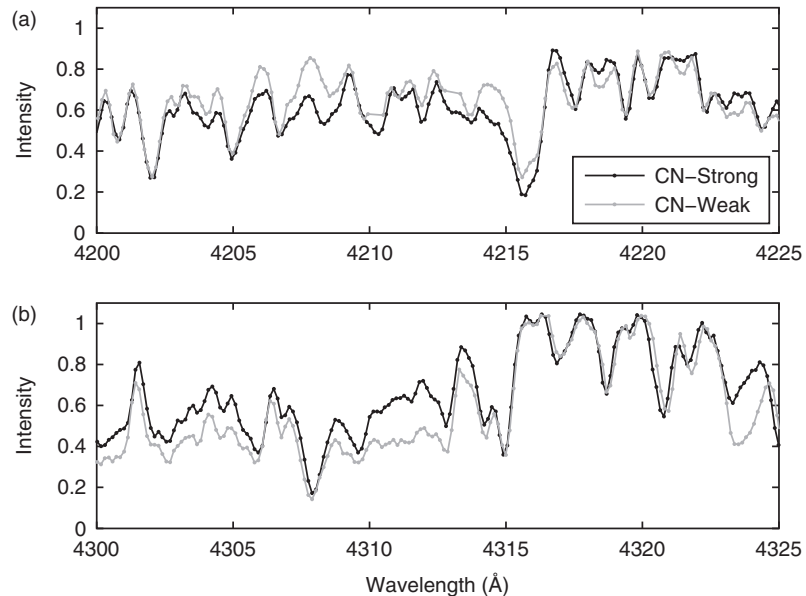

Figure 4 Comparison of a CN-weak star (grey, Lee 4506, $\delta \mathrm{C}_{2011}=0.050$ ) with a CN-strong star (black, Pal 578, $\left.\delta C_{2011}=0.164\right)$ of similar $T_{\text {eff }}$ and $\log g$ from the 47 Tuc stellar survey. (a) CN bandhead at $4216 \AA$. (b) G Band.

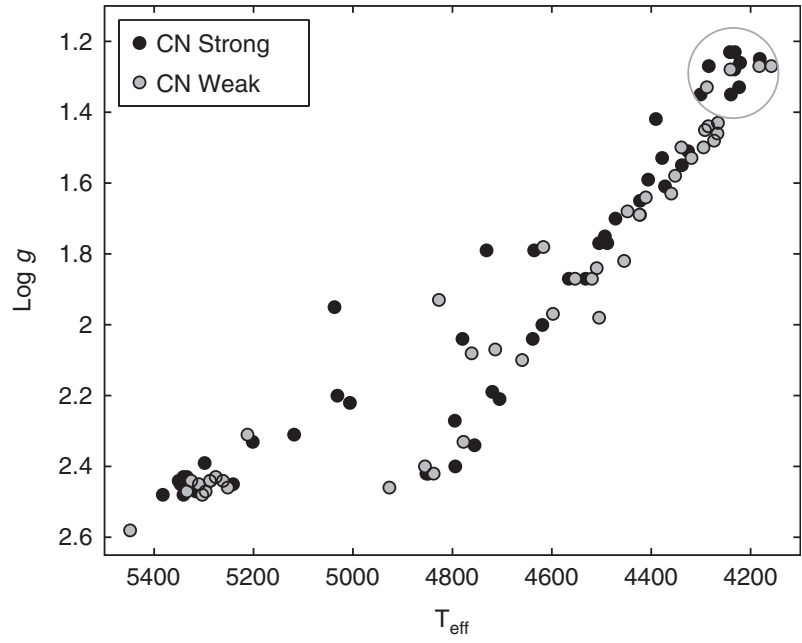

Figure $5 T_{\text {eff }}-\log g$ space for $\mathrm{CN}$-weak (grey circle) and $\mathrm{CN}$-strong (black circle) pairs based on $V-K$ photometry. The circle encompasses Lee 2525-like stars.

better reflects the precision of the data (cf. NF79; PF84). The $\mathrm{CN}$ bimodality is seen as two peaks, one for the $\mathrm{CN}$-weak stars at $\delta \mathrm{C}_{2011} \sim 0.054$ and the other for the $\mathrm{CN}$-strong stars at $\delta \mathrm{C}_{2011} \sim 0.166$. These values are the mean $(\mu)$ values for each population that were determined by the application of a simple Gaussian mixture model. The model determined $\sigma=0.055$ for both populations in mixing proportions of 0.48 for the $\mathrm{CN}$-weak population, and 0.52 for the $\mathrm{CN}$-strong population. The difference in the means, $\Delta \mu=0.112$, is slightly greater than $2 \sigma=0.11$ which is the minimum limit necessary to detect two populations in a single dataset (Reschenhofer 2001). Hence two populations in $\delta \mathrm{C}_{2011}$ exist within this dataset.

The CN-CH anti-correlation is a well-known feature of 47 Tuc and other globular clusters (Cannon et al. 1998). Figure 4 compares a $\mathrm{CN}$-weak and a $\mathrm{CN}$-strong star of similar $T_{\text {eff }}$ and $\log g$. The $\mathrm{CN}$ bandhead (degrading to the blue from $\sim 4216 \AA$ ) is distinctly different between the $\mathrm{CN}$ weak and $\mathrm{CN}$-strong stars. At the $\mathrm{CH}$ band the $\mathrm{CN}$-strong 
star has a weak $\mathrm{CH}$ band, corresponding to carbon depletion and nitrogen enrichment from the $\mathrm{CN}$ band.

\section{Atmospheric Parameters}

The stellar atmospheric models for each star were based on the effective temperature $\left(T_{\text {eff }}\right)$ and surface gravity $(\log g)$ values derived from the $V-K$ photometry and

Table 3. The stellar parameters, $T_{\text {eff }}$ and $\log g$, were calculated for each star based on $V-K$ photometry for 13 stars of similar stellar parameters

\begin{tabular}{lrcccr}
\hline Star ID $^{\mathrm{a}}$ & $\delta_{2011}^{\mathrm{b}}$ & $\delta C_{1979}^{\mathrm{a}}$ & Ref. $^{\mathrm{a}}$ & $T_{\text {eff }}$ & $\log g$ \\
\hline Pa1502 & -0.04 & 0.02 & PF84 & 4158 & 1.27 \\
Lee2306 & 0.00 & 0.05 & NF79 & 4288 & 1.33 \\
Lee3622 & 0.01 & 0.03 & NF79 & 4183 & 1.27 \\
Lee4628 & 0.04 & 0.05 & NF79 & 4241 & 1.28 \\
Lee2525 & 0.08 & 0.10 & NF79 & 4232 & 1.23 \\
W66 & 0.08 & - & - & 4181 & 1.25 \\
Lee5703 & 0.13 & 0.02 & NF79 & 4221 & 1.26 \\
Lee1513 & 0.14 & 0.21 & NF79 & 4242 & 1.23 \\
W139 & 0.15 & - & - & 4223 & 1.33 \\
Pa1262 & 0.17 & 0.25 & PF84 & 4240 & 1.35 \\
W164 & 0.17 & - & - & 4300 & 1.35 \\
Pa1661 & 0.20 & 0.22 & PF84 & 4233 & 1.28 \\
Lee1747 & 0.22 & 0.25 & NF79 & 4284 & 1.27 \\
& & & Mean & 4233 & 1.28 \\
& & & $\sigma$ & \pm 44 & \pm 0.09 \\
\hline
\end{tabular}

${ }^{\mathrm{a}} \mathrm{Star}$ ID, $\delta \mathrm{C}_{1979}$ taken from NF79 and PF84.

${ }^{\mathrm{b}} \mathrm{\delta C}_{2011}$ from this study. using the relationships in Alonso et al. (1999). Comparison between $\mathrm{CN}$-weak and $\mathrm{CN}$-strong stars is ideally investigated using stars at similar $T_{\text {eff }}$ and $\log g$. These are shown in $T_{\text {eff }}-\log g$ space in Figure 5 and contain many pairs on the RGB, HB and AGB.

The group of 13 stars (see circle in Figure 5) at $T_{\text {eff }} \approx$ $4200 \mathrm{~K}$ and $\log g \approx 1.3$ includes the star Lee 2525 , for which a high-resolution analysis was carried out by Worley et al. (2009). The stars in this group have approximately the same stellar parameters and provide a sample of comparable stars at different $\mathrm{CN}$ strengths. The stellar designations, $\mathrm{CN}$ excess, photometry and stellar parameters for these stars are listed in Table 3, and are analysed in the remainder of this paper.

\subsection{Lee 2525}

Lee 2525 is a 47 Tuc giant star that has been observed in several studies. It has been singled out as a linking star in two previous studies (Wylie et al. 2006; Brown \& Wallerstein 1992). It has been observed in three separate datasets: SALT PV RSS medium-resolution observations of 11 stars in 47 Tuc (Worley et al. 2008); AAOmega 47 Tuc medium-resolution survey (this study); and highresolution observation of Lee 2525 on the SSO $2.3 \mathrm{~m}$ telescope (Worley et al. 2009). Figure 6 compares the spectra of Lee 2525 from all three studies in the regions of the light and heavy element spectral features. For comparison, the SSO high-resolution spectrum was convolved
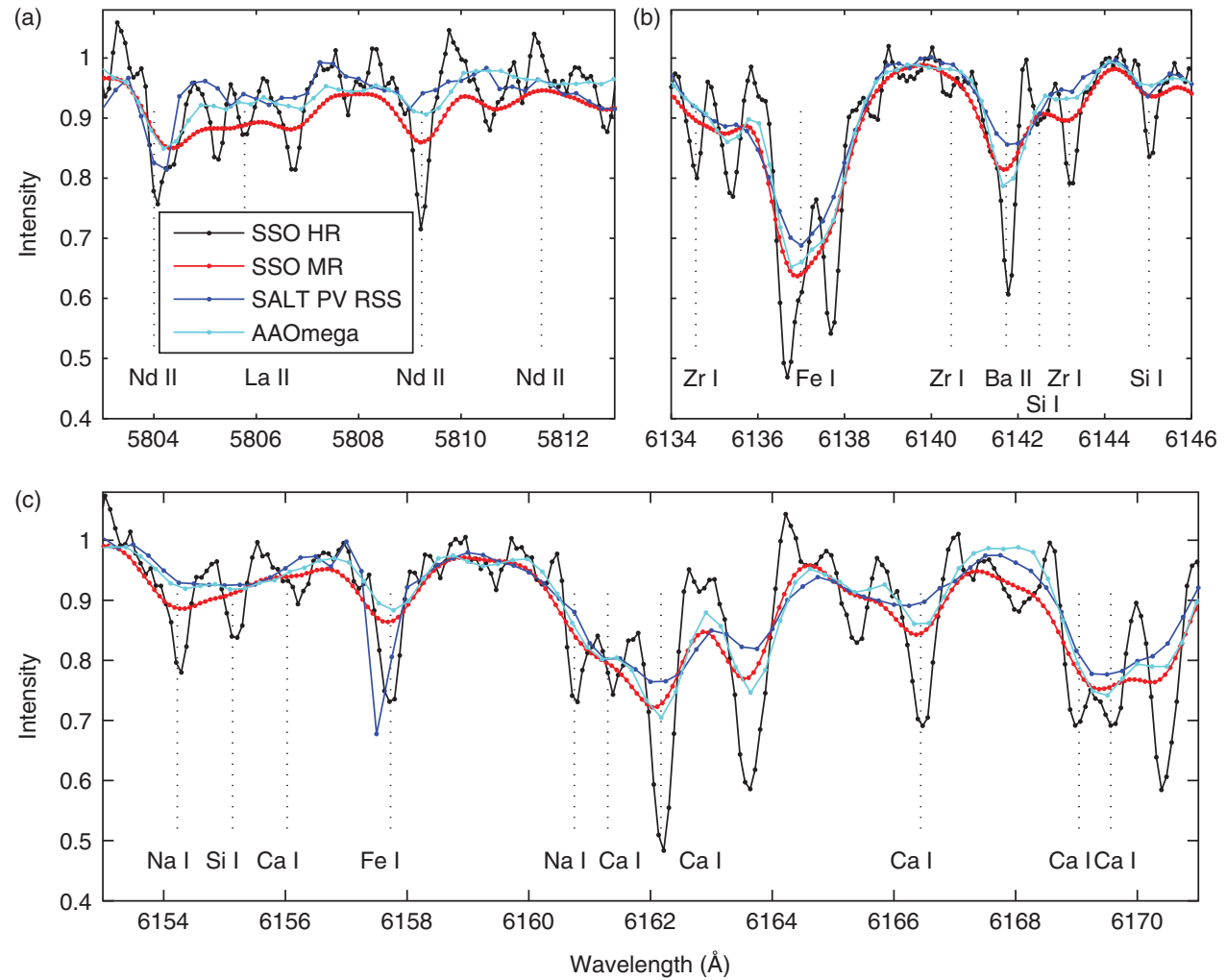

Figure 6 Comparison of the Lee 2525 spectra observed using RSS on SALT (blue), the SSO 2.3-m telescope (black), and AAOmega on the AAT (cyan). Also included is the convolution of the high-resolution spectra to a resolution comparable to the AAOmega observation (red). (a) Nd II and La II features in the 5800 A region. (b) Si I, Fe I, Ba II and Zr I features in the 6140 A region. (c) Na I, Si I, Ca I and Fe I features in the 6160 A region. 

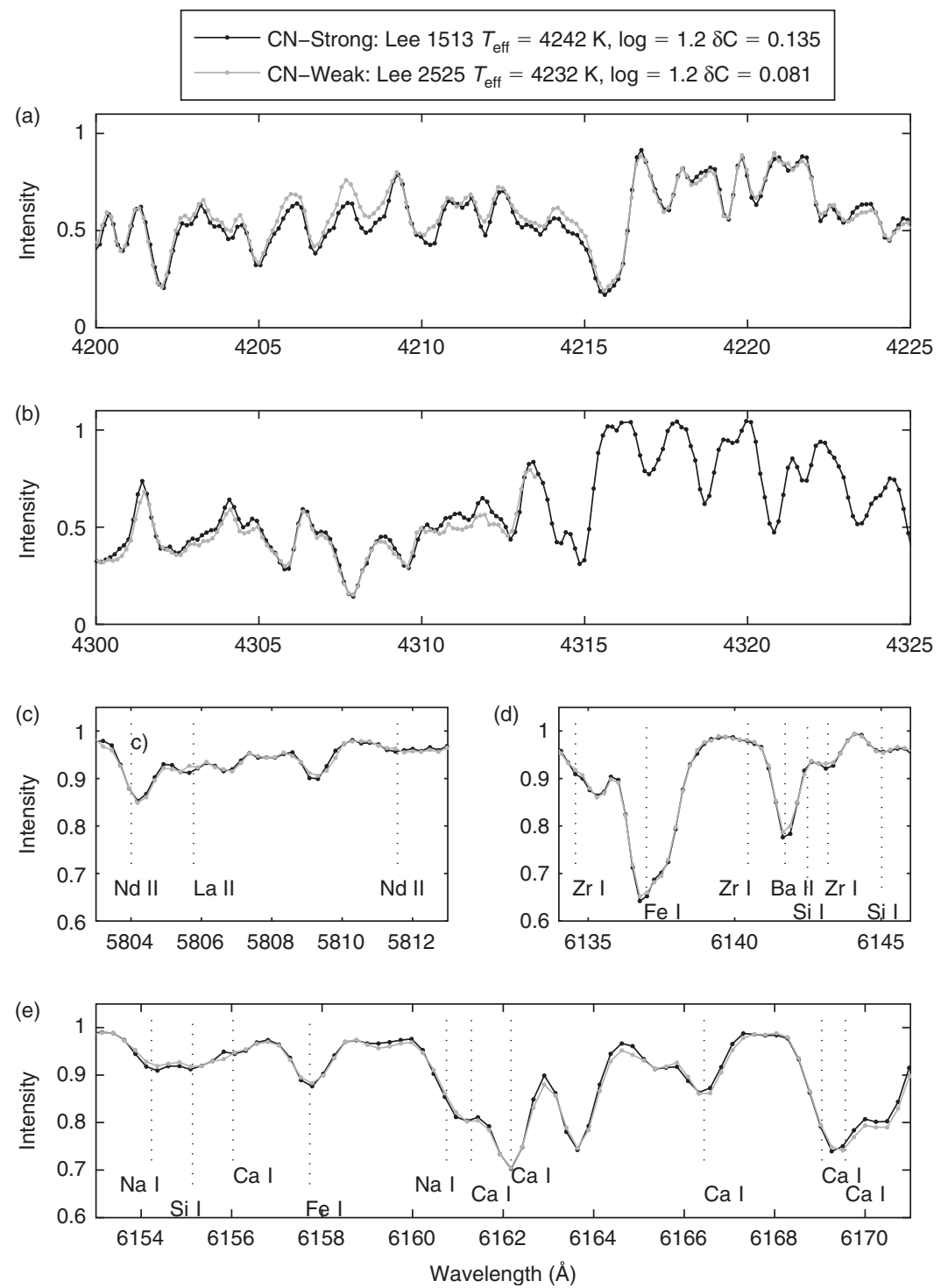

Figure 7 Comparison of Lee 2525 (grey) and Lee 1513 (black) spectra. (a) CN band, (b) CH band, (c) Nd II and La II, (d) Si I, Fe I, Ba II and Zr I, (e) Na I, Si I, Ca I and Fe I.

to a resolution comparable to the AAOmega data in this study.

The Lee 2525 spectrum observed on RSS is at a lower resolution than the AAOmega spectra. The features in common between the high- and medium-resolution spectra agree in terms of their relative line depths. Abundances derived by spectrum synthesis were obtained for the light and heavy elements for each spectrum, including the convolved SSO spectrum, and are discussed in the next section.

In NF79, Lee 2525 was found to be a CN-weak star with $\delta \mathrm{C}_{1979}=0.10$. Its $\mathrm{CN}$-strong pair was Lee 1513 which had a $\mathrm{CN}$ excess of $\delta \mathrm{C}_{1979}=0.21$ (Brown \& Wallerstein 1992). This pairing holds in the current analysis where Lee 2525 was designated as $\mathrm{CN}$-weak $\left(\delta \mathrm{C}_{2011}=0.08\right)$ and Lee 1513 as $\mathrm{CN}$-strong $\left(\delta \mathrm{C}_{2011}=\right.$ 0.14 ), although not as strong as in the previous study.

Figure 7 compares the spectra from these two stars, Lee 2525 and Lee 1513. The two spectra are strikingly similar in all regions except the $\mathrm{CN}$ where the smaller $\delta \mathrm{C}$ in Lee 2525 can be discerned. The remaining spectral regions are also almost an exact match, except that the $\mathrm{Na}$ feature at $6154 \AA$ is slightly enhanced in the $\mathrm{CN}$-strong spectrum relative to the $\mathrm{CN}$-weak spectrum.

Figure 8 compares two stars that are well separated in $\mathrm{CN}$ excess value but have similar overall stellar parameters. Pal 262 is a CN-strong star with $\delta \mathrm{C}_{2011}=0.17$, while Lee 2603 is a CN-weak star with $\delta \mathrm{C}_{2011}=0.00$. The differences in $\mathrm{CN}$ and $\mathrm{CH}$ strength are particularly distinct. What also becomes apparent is the difference in the line strength of the two Na I lines at $6154 \AA$ and $6160 \AA$. The CN-strong star was considerably stronger in $\mathrm{Na}$ I lines than the $\mathrm{CN}$-weak star. The correlation of $\mathrm{Na}$ I with $\mathrm{CN}$ strength has been noted in previous studies (Gratton et al. 2004; Cottrell \& Da Costa 1981), and will be analysed in Section 5.1. The remaining features, in particular the $s$-process element features, show no distinct difference in line strength between these stars. 

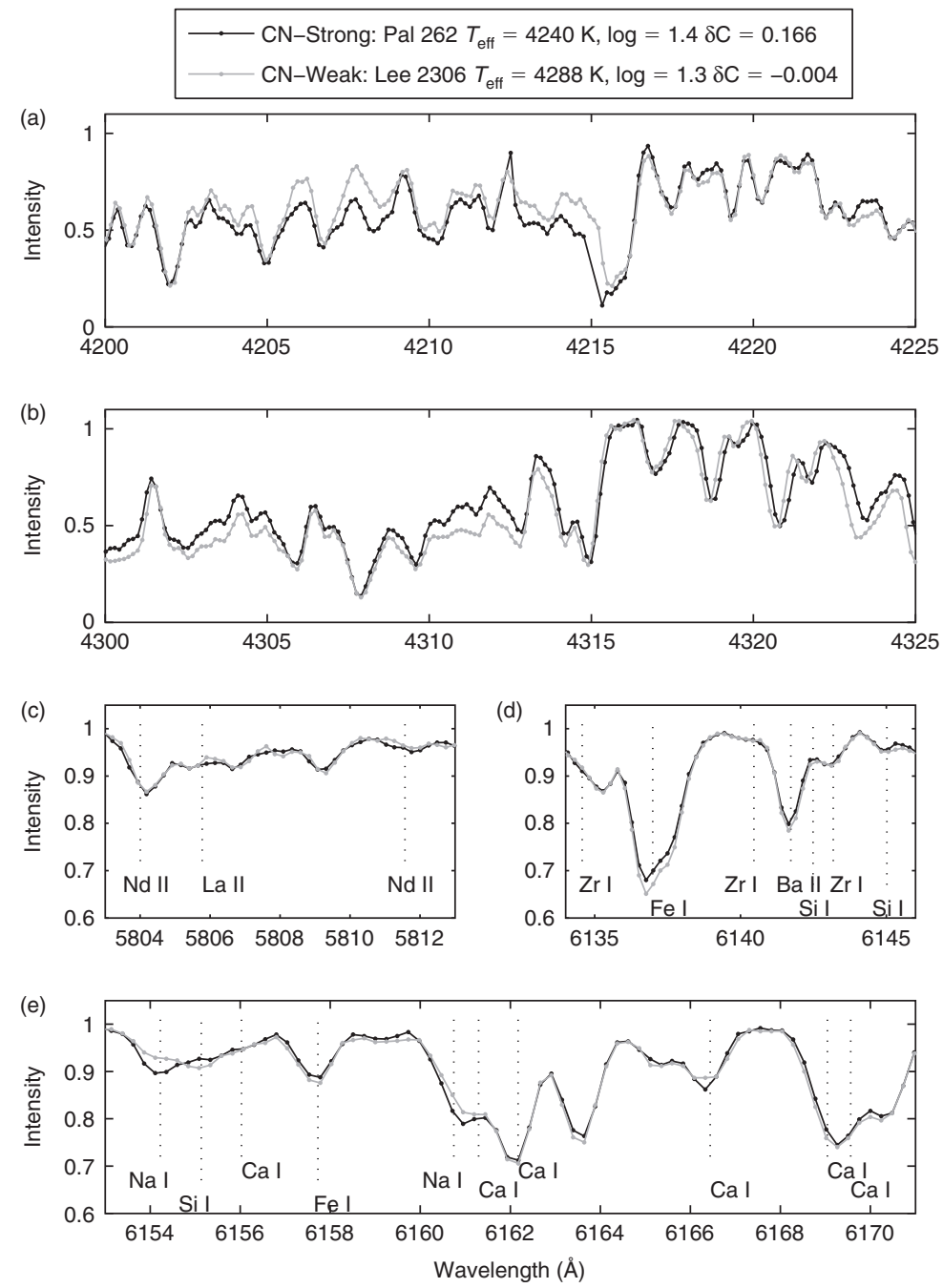

Figure 8 As for Figure 7, but comparing the spectra of Pal 262 (black) and Lee 2603 (grey).

\section{Lee 2525 Sample Analysis}

The subset of stars at $T_{\text {eff }} \approx 4200 \mathrm{~K}$ and $\log g \approx 1.3$ was used to investigate the abundances of the light and heavy elements in 47 Tuc stars. In the high-resolution analysis of Lee 2525 (Worley et al. 2009) the stellar atmospheric model for Lee 2525 was determined to have $T_{\text {eff }}=4225 \mathrm{~K}$, $\log g=1.2, \xi=1.8 \mathrm{~km} \mathrm{~s}^{-1}$ and $[\mathrm{Fe} / \mathrm{H}]=-0.70 \mathrm{dex}$. This model was used in the following abundance analysis of the medium-resolution observation of Lee 2525. It was subsequently used as the model for all the remaining stars in the subset defined in Table 3 as it is within $1 \sigma(10 \mathrm{~K}$ and $0.1 \mathrm{dex}$ ) of the mean $T_{\text {eff }}$ and $\log g$ for all of these stars.

In order to make a direct comparison, the highresolution SSO Lee 2525 spectrum was convolved to the same resolution as the AAOmega spectrum and an abundance analysis was carried out. The results from that analysis and a spectrum synthesis analysis of the highresolution Lee 2525 spectrum are also listed in Table $4 .{ }^{4}$

\footnotetext{
${ }^{4}$ From this section onwards the definition of the bracket notation $[\mathrm{X} / \mathrm{H}]$ or $[\mathrm{X} / \mathrm{Fe}]$ is $\log _{10}(\mathrm{X} / \mathrm{H})_{\star}-\log _{10}(\mathrm{X} / \mathrm{H})_{\text {Arcturus }}$ or $\log _{10}(\mathrm{X} / \mathrm{Fe})_{\star}-$ $\log _{10}(\mathrm{X} / \mathrm{Fe})_{\text {Arcturus }}$.
}

There is reasonable agreement in the abundance for $\mathrm{Fe}$ for all three sets of results with a similar level of uncertainty $(\sim 0.08$ dex $)$. For the light elements, the convolved SSO spectrum produced the largest spread in values ( $\sim 0.2$ dex) between the lines for each of $\mathrm{Na}, \mathrm{Si}$ and $\mathrm{Ca}$, while for the other two spectra there were smaller uncertainties in each $(\leq 0.11 \mathrm{dex})$. Clearly the information retained in a simple Gaussian convolution does not necessarily match observations at the resolution to which the convolution was made.

For each of the light and heavy elements there are discrepancies between the abundance determinations of these different Lee 2525 spectra, which is an example of how the degradation of spectra to lower resolution implies a different abundance of an element. Also the highresolution spectrum of Lee 2525 had SNR $\sim 50$ so features that are due to the noise are likely to have contaminated the shape of the spectral features in the convolution. There was much better agreement between abundances derived from the Arcturus high-resolution and convolved spectra (see Table 2). A high-SNR, highresolution observation of Lee 2525 would provide the basis for a more consistent comparative analysis. 
Table 4. Elemental abundances derived for Lee 2525 from the medium-resolution AAOmega spectrum (column 3), from the convolved high-resolution SSO 2.3-m spectrum (column 4) and from the high-resolution analysis (column 5), all calculated differentially with respect to the respective Arcturus MR or HR abundances (errors with changes in stellar parameters for the AAOmega medium resolution spectrum are shown)

\begin{tabular}{|c|c|c|c|c|c|c|c|}
\hline & $\begin{array}{l}\text { Instrument } \\
\text { Resolution }\end{array}$ & $\begin{array}{l}\text { AAOmega } \\
\text { Medium }\end{array}$ & $\begin{array}{l}\text { SSO } 2.3 \mathrm{~m} \\
\text { Convolved }\end{array}$ & $\begin{array}{c}\text { SSO } 2.3 \mathrm{~m} \\
\text { High }\end{array}$ & \multicolumn{3}{|c|}{$\Delta[\mathrm{Fe} / \mathrm{H}]$} \\
\hline & $\lambda(\AA)$ & {$[\mathrm{Fe} / \mathrm{H}]$} & {$[\mathrm{Fe} / \mathrm{H}]$} & {$[\mathrm{Fe} / \mathrm{H}]$} & $\begin{array}{c}\Delta T_{\text {eff }} \\
+100 \mathrm{~K}\end{array}$ & $\begin{array}{c}\Delta \log g \\
0.5\end{array}$ & $\begin{array}{r}\Delta \xi \\
0.5\end{array}$ \\
\hline $\mathrm{Fe} I$ & 6136.62 & -0.13 & -0.03 & 0.08 & 0.03 & 0.10 & -0.33 \\
\hline $\mathrm{Fe} \mathrm{I}$ & 6147.83 & 0.02 & 0.11 & -0.04 & -0.08 & 0.15 & -0.13 \\
\hline \multirow[t]{3}{*}{$\mathrm{Fe} \mathrm{I}$} & 6157.73 & -0.02 & 0.10 & 0.10 & -0.03 & 0.11 & -0.26 \\
\hline & $\langle[\mathrm{Fe} / \mathrm{H}]\rangle$ & -0.04 & 0.06 & 0.05 & -0.05 & 0.13 & -0.20 \\
\hline & $\sigma$ & 0.08 & 0.08 & 0.08 & 0.13 & 0.16 & 0.21 \\
\hline $\mathrm{X}$ & & {$[\mathrm{X} / \mathrm{Fe}]$} & {$[\mathrm{X} / \mathrm{Fe}]$} & {$[\mathrm{X} / \mathrm{Fe}]$} & & $\Delta[\mathrm{X} / \mathrm{Fe}]$ & \\
\hline $\mathrm{NaI}$ & 6154.23 & 0.00 & 0.23 & 0.12 & 0.06 & -0.02 & -0.09 \\
\hline \multirow[t]{3}{*}{$\mathrm{Na} \mathrm{I}$} & 6160.75 & -0.08 & -0.10 & 0.15 & 0.08 & 0.00 & -0.12 \\
\hline & $\langle[\mathrm{Na} / \mathrm{Fe}]\rangle$ & -0.04 & 0.06 & 0.14 & 0.07 & -0.01 & -0.11 \\
\hline & $\sigma$ & 0.06 & 0.23 & 0.02 & 0.01 & 0.01 & 0.02 \\
\hline Si I & 6131.57 & 0.09 & 0.06 & 0.10 & -0.02 & 0.13 & 0.01 \\
\hline Si I & 6131.85 & 0.08 & 0.05 & 0.09 & 0.00 & 0.18 & 0.03 \\
\hline Si I & 6145.02 & 0.07 & 0.22 & 0.06 & -0.01 & 0.17 & -0.04 \\
\hline \multirow[t]{3}{*}{ Si I } & 6155.13 & -0.12 & -0.31 & -0.04 & -0.03 & 0.14 & -0.08 \\
\hline & $\langle[\mathrm{Si} / \mathrm{Fe}]\rangle$ & 0.03 & 0.00 & 0.05 & -0.02 & 0.16 & -0.02 \\
\hline & $\sigma$ & 0.01 & 0.22 & 0.06 & 0.03 & 0.03 & 0.07 \\
\hline $\mathrm{Ca} \mathrm{I}$ & 6156.02 & -0.14 & -0.21 & -0.14 & 0.05 & 0.03 & -0.07 \\
\hline \multirow[t]{3}{*}{$\mathrm{Ca} \mathrm{I}$} & 6166.44 & 0.01 & 0.07 & -0.11 & 0.13 & 0.09 & -0.23 \\
\hline & $\langle[\mathrm{Ca} / \mathrm{Fe}]\rangle$ & -0.07 & -0.07 & -0.13 & 0.09 & 0.06 & -0.15 \\
\hline & $\sigma$ & 0.11 & 0.20 & 0.02 & 0.06 & 0.04 & 0.11 \\
\hline Zr I & 6143.18 & 0.02 & 0.42 & 0.28 & 0.15 & 0.05 & -0.10 \\
\hline $\mathrm{Ba}$ II & 6141.73 & 0.26 & -0.14 & 0.00 & 0.00 & 0.17 & -0.60 \\
\hline La II & 5805.77 & -0.03 & 0.45 & 0.21 & 0.08 & -0.08 & -0.08 \\
\hline Nd II & 5811.57 & -0.20 & -0.06 & 0.00 & 0.10 & 0.03 & 0.03 \\
\hline
\end{tabular}

Table 5. Elemental abundances with respect to the Sun derived for the convolved high-resolution Arcturus atlas and elemental abundances with respect to the Arcturus values for the 13 Lee $\sim 2525$-like stars in the 47 Tuc medium-resolution survey, inferred using the Lee 2525 stellar atmospheric model (the mean abundances and standard deviations for the entire 47 Tuc sample are also listed)

\begin{tabular}{lccccccrrr}
\hline Star & Arcturus & Pa1502 & Lee2306 & Lee3622 & Lee4628 & Lee2525 & W66 & Lee5703 & Lee1513 \\
\hline$\xi$ & 1.5 & 1.8 & 1.8 & 1.5 & 1.8 & 1.8 & 1.8 & 1.8 & 2.0 \\
$\delta \mathrm{C}_{2011}$ & -0.06 & -0.04 & 0.00 & 0.01 & 0.04 & 0.08 & 0.08 & 0.13 & 0.14 \\
{$[\mathrm{Fe} / \mathrm{H}]$} & -0.53 & -0.20 & -0.14 & -0.14 & -0.13 & -0.14 & -0.15 & -0.14 & -0.15 \\
{$[\mathrm{Na} / \mathrm{Fe}]$} & 0.06 & -0.06 & -0.21 & -0.13 & -0.18 & -0.10 & 0.06 & -0.06 & -0.02 \\
{$[\mathrm{Si} / \mathrm{Fe}]$} & 0.26 & -0.06 & 0.02 & -0.08 & 0.03 & 0.03 & -0.08 & -0.02 & 0.01 \\
{$[\mathrm{Ca} / \mathrm{Fe}]$} & 0.25 & 0.05 & -0.14 & 0.03 & -0.03 & -0.07 & -0.01 & -0.07 & -0.12 \\
{$[\mathrm{Zr} / \mathrm{Fe}]$} & -0.12 & 0.32 & 0.07 & 0.22 & 0.22 & 0.02 & 0.27 & 0.09 & 0.10 \\
{$[\mathrm{Ba} / \mathrm{Fe}]$} & 0.04 & 0.21 & 0.24 & 0.29 & 0.31 & 0.26 & 0.21 & 0.36 & 0.29 \\
{$[h s / l s]$} & 0.16 & -0.11 & 0.17 & 0.07 & 0.09 & 0.24 & -0.06 & 0.27 \\
& & & & & & & & 0.19 \\
$\mathrm{Star}$ & $\mathrm{W} 139$ & $\mathrm{~Pa} 262$ & $\mathrm{~W} 164$ & $\mathrm{~Pa} 1661$ & Lee1747 & & Sample & Statistics \\
$\xi$ & 1.8 & 1.5 & 1.8 & 1.8 & 1.8 & $\langle\xi\rangle$ & 1.8 & 0.13 \\
$\delta \mathrm{C}_{2011}$ & 0.15 & 0.17 & 0.17 & 0.20 & 0.22 & $\langle\delta \mathrm{C} 2011\rangle$ & 0.10 & 0.09 \\
{$[\mathrm{Fe} / \mathrm{H}]$} & -0.13 & -0.16 & -0.16 & -0.12 & -0.11 & $\langle[\mathrm{Fe} / \mathrm{H}]\rangle$ & -0.14 & 0.02 \\
{$[\mathrm{Na} / \mathrm{Fe}]$} & -0.08 & 0.25 & 0.02 & 0.02 & 0.17 & $\langle[\mathrm{Na} / \mathrm{Fe}]\rangle$ & -0.02 & 0.13 \\
{$[\mathrm{Si} / \mathrm{Fe}]$} & 0.04 & -0.01 & -0.08 & 0.00 & 0.01 & $\langle[\mathrm{Si} / \mathrm{Fe}]\rangle$ & -0.01 & 0.04 \\
{$[\mathrm{Ca} / \mathrm{Fe}]$} & -0.14 & -0.03 & -0.11 & -0.09 & -0.12 & $\langle[\mathrm{Ca} / \mathrm{Fe}]\rangle$ & -0.06 & 0.06 \\
{$[\mathrm{Zr} / \mathrm{Fe}]$} & 0.12 & 0.12 & 0.12 & 0.07 & 0.07 & $\langle[\mathrm{Zr} / \mathrm{Fe}]\rangle$ & 0.14 & 0.09 \\
{$[\mathrm{Ba} / \mathrm{Fe}]$} & 0.26 & 0.31 & 0.36 & 0.26 & 0.26 & $\langle[\mathrm{Ba} / \mathrm{Fe}]\rangle$ & 0.28 & 0.05 \\
{$[h s / l s]$} & 0.14 & 0.19 & 0.24 & 0.19 & 0.19 & $\langle[h s / l s]\rangle$ & 0.14 & 0.11 \\
\hline
\end{tabular}



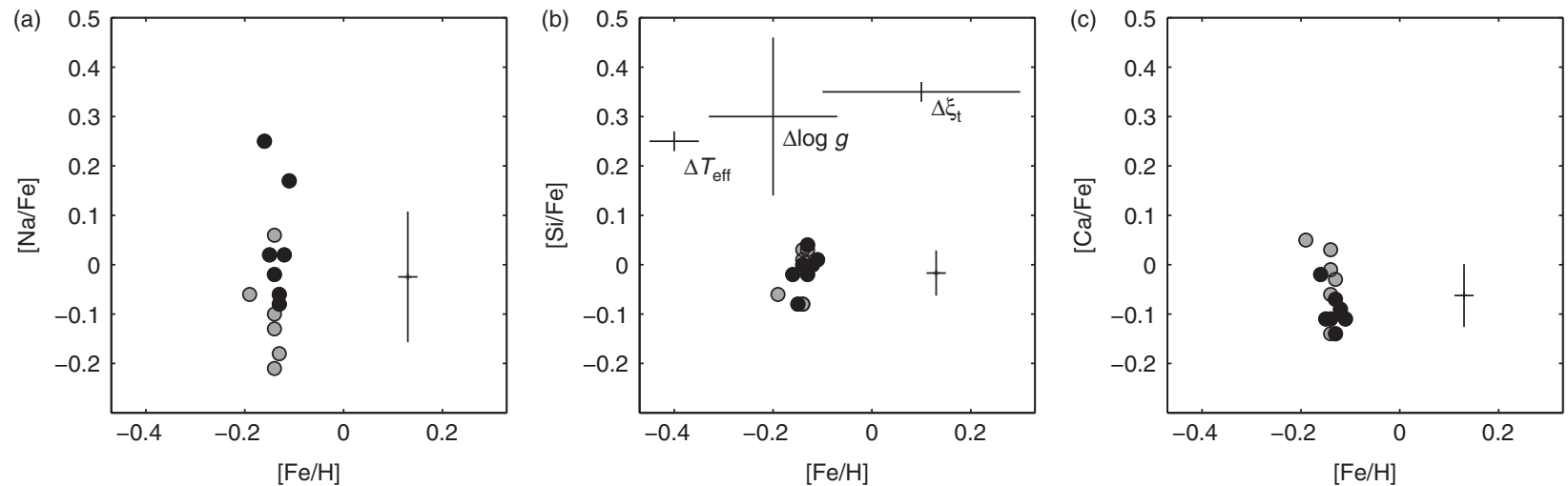

Figure 9 The element abundance ratios $[\mathrm{X} / \mathrm{Fe}]$ calculated with respect to the medium-resolution abundance analysis of Arcturus for (a) Na, (b) $\mathrm{Si}$ and (c) $\mathrm{Ca}$, against $[\mathrm{Fe} / \mathrm{H}]$ for each of the 13 'Lee 2525-like' stars. Grey circles are $\mathrm{CN}$-weak stars and black circles are $\mathrm{CN}$-strong. The sample mean and standard deviation for each is also shown. The systematic uncertainties from Table 4 are illustrated for $[\mathrm{Si} / \mathrm{Fe}]$.

The error analysis in Table 4 shows changes in the elemental abundances on the order of 0.1 dex for changes in the stellar parameters based on the intervals in the stellar model grid.

With regard to the $s$-process element abundances, the spectral features for $\mathrm{La}$ and $\mathrm{Nd}$ are heavily blended at this resolution (see Figure 6) and only the Ba line at $6141.73 \AA$ and the $\mathrm{Zr}$ line at $6143.18 \AA$ looked sufficiently distinct for analysis. Abundances were determined for $\mathrm{La}$ and $\mathrm{Nd}$ for the Lee 2525 spectra, but the features were deemed to be too blended $(R \sim 6500)$ for analysis in the remaining survey stars.

Following this analysis of the medium-resolution Lee 2525 spectra the other stars in Table 3 were analysed for their elemental abundances. As noted earlier, the model determined for Lee 2525 was used in the analysis of the spectra for each of these stars. The only parameter that was varied was the microturbulence $\left(\xi_{t}\right)$ as it was clear for four of the stars that the Lee 2525 microturbulence value was not a good fit. Table 5 lists the stellar parameters, CN excess and elemental abundances for each of the thirteen stars in this subset. The $[h s / l s]$ ratio is included as the difference between the $\mathrm{Ba}$ (heavy s-process) and $\mathrm{Zr}$ (light $s$-process) abundances.

\subsection{Light Elemental Abundances}

The light-elemental abundance ratios with respect to Arcturus are compared with $[\mathrm{Fe} / \mathrm{H}]$ in Figure 9. The $[\mathrm{Si} / \mathrm{Fe}]$ and $[\mathrm{Ca} / \mathrm{Fe}]$ abundance ratios each have a small spread (of $<0.2 \mathrm{dex}$ ) for this sample, indicating that there is a homogeneous abundance distribution for these two elements. The systematic uncertainties with associated changes in $T_{\text {eff }} \log g$ and $\xi_{t}$ presented in Table 4 are shown for $[\mathrm{Si} / \mathrm{Fe}]$ and $[\mathrm{Fe} / \mathrm{H}]$ in Figure $9 \mathrm{~b}$ to illustrate the range in values with changes in stellar parameters. The spread in the $[\mathrm{Na} / \mathrm{Fe}]$ far exceeds what can be expected from the corresponding systematic uncertainties of $\sim 0.2$ dex (Table 4). This implies that the range in $[\mathrm{Na} / \mathrm{Fe}]$ is real.

Furthermore, the $\mathrm{Na}-\mathrm{CN}$ strength correlation is well documented in globular cluster stars (Cottrell \& Da

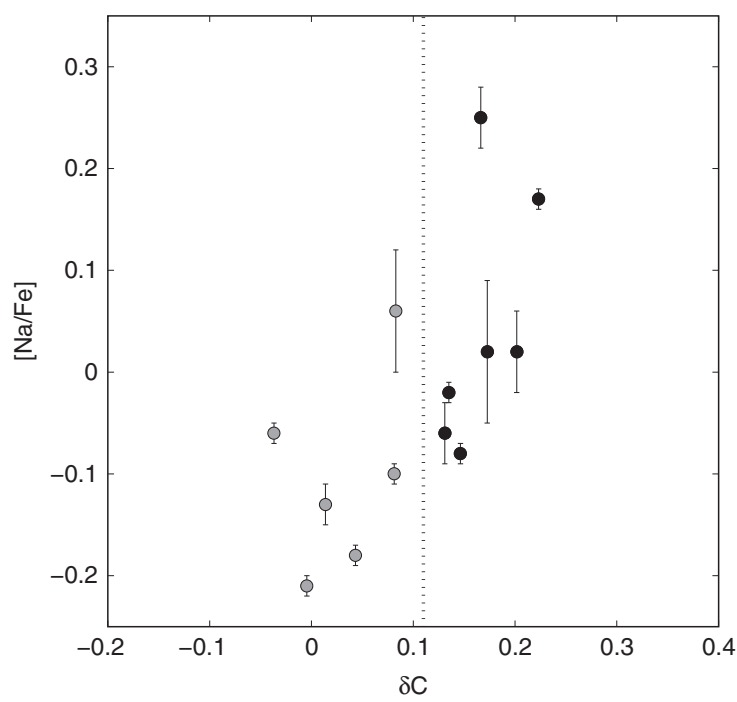

Figure $10[\mathrm{Na} / \mathrm{Fe}]$ with respect to Arcturus against $\delta C_{2011}$ for each of the thirteen Lee 2525-like stars. The dotted line shows the threshold at 0.11 . Grey circles are $\mathrm{CN}$-weak stars and black circles are $\mathrm{CN}$-strong.

Costa 1981). Figure 10 compares the $[\mathrm{Na} / \mathrm{Fe}]$ abundance ratio with $\mathrm{CN}$ strength $\left(\delta \mathrm{C}_{2011}\right)$. There is a clear correlation, with the increasing enrichment in $\mathrm{Na}$ corresponding to increasing $\mathrm{CN}$ strength. The trend seems continuous rather than attributable to two distinct populations. Of the stars in the Lee 2525 -like sample, 10 have previously determined $\delta \mathrm{C}$ values, one of which is Lee 5703 for which the $\delta \mathrm{C}_{2011}$ differed above $2 \sigma$ from the $\delta \mathrm{C}_{1979}$ value. However, is it not one of the outliers in Figure 10, nor for the remaining abundance determinations, implying that the current analysis of that star is reliable.

\subsection{Heavy Elemental Abundances}

Figure 11 shows the abundance ratios for each of $[\mathrm{Zr} / \mathrm{Fe}]$ and $[\mathrm{Ba} / \mathrm{Fe}]$ against $[\mathrm{Fe} / \mathrm{H}]$ for the Lee 2525 -like sample. In Figure 10 there is a distinct spread in the values for 

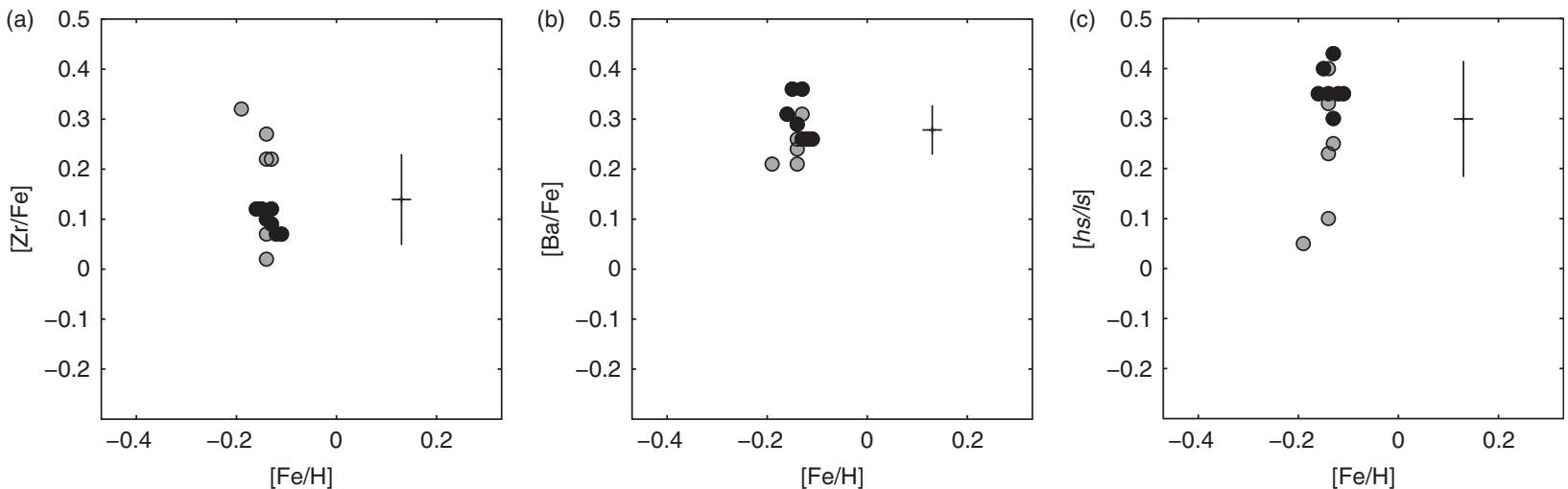

Figure 11 The abundances of the $s$-process elements with respect to Arcturus, (a) $\mathrm{Zr}$ and (b) Ba, against $[\mathrm{Fe} / \mathrm{H}]$ for the thirteen Lee 2525 -like stars in the 47 Tuc medium-resolution survey with sample mean and standard deviation. (c) The ratio of [hs/ls] against [Fe/H] for the sample. Grey circles are $\mathrm{CN}$-weak stars and black circles are $\mathrm{CN}$-strong.

$[\mathrm{Zr} / \mathrm{Fe}]$. However the $\mathrm{Zr}$ feature used in this analysis (see Figure 7) is small and quite blended at this resolution so the larger uncertainly is not unexpected. There is however a very small spread in the $[\mathrm{Ba} / \mathrm{Fe}]$ abundance ratios (Figure 11) that is similar to that for $[\mathrm{Si} / \mathrm{Fe}]$ in Figure 9. The $\mathrm{Ba}$ feature used here is very senstive to $\xi_{\mathrm{t}}$. However the small spread indicates that the adjustment of the $\xi_{t}$ values resulted in consistency between the $\mathrm{Ba}$ and $\mathrm{Fe}$ abundances.

Figure 11 shows the distribution of the $[h s / l s]$ ratio against $[\mathrm{Fe} / \mathrm{H}]$ for this sample. The spread in $[\mathrm{Fe} / \mathrm{H}]$ is very small at 0.02 dex while the larger spread in the $[h s / l s]$ values can mainly be attributed to the spread in the $\mathrm{Zr}$ abundances; hence, they are subject to large systematic uncertainties. The sample mean value of $\langle[h s / l s]\rangle=$ $0.14 \pm 0.11 \mathrm{dex}$ is slightly higher than some recent analysis of high-resolution spectra of 47 Tuc giant stars, $[h s / l s]=-0.13 \pm 0.05 \mathrm{dex}$ (Worley et al. 2010). Both of these values are indicative of values for stars at this metallicity (Busso et al. 2001). This analysis shows that to properly survey the weak $s$-process spectral features a higher resolution is needed.

\section{Conclusion}

The medium-resolution survey of 47 Tuc stars has provided a good sample with which to investigate several aspects of chemical abundances in GCs. The survey sample clearly shows the $\mathrm{CN}-\mathrm{CH}$ anti-correlation which is well documented for GCs (Norris \& Freeman 1979; Cannon et al. 1998, 2003; Briley et al. 2004). The bimodal distribution of $\mathrm{CN}$-weak and $\mathrm{CN}$-strong stars is also evident in the sample. The current measurement of the $\mathrm{CN}$ indices of these stars were in reasonable agreement with the previous studies of NF79 and PF84.

The grouping of stars about Lee 2525 provided a unique sample with which to investigate abundance variations for stars of very similar stellar parameters. To complement the $\mathrm{CN}$ indices, the medium-resolution spectra were analysed for light and heavy element abundances using spectrum synthesis techniques. This preliminary set of stars, and their representative star, Lee 2525, provided a link to high-resolution abundance analyses of 47 Tuc giant stars in previous studies (Brown \& Wallerstein 1992; Wylie et al. 2006; Worley et al. 2008, 2010).

Relative to Arcturus, the abundance analyses for these stars give a strong indication for a homogenous distribution of $\mathrm{Fe}(\langle[\mathrm{Fe} / \mathrm{H}]\rangle=-0.14 \pm 0.02 \mathrm{dex}), \mathrm{Si}$ $(\langle[\mathrm{Si} / \mathrm{Fe}]\rangle=-0.01 \pm 0.04 \mathrm{dex})$ and $\mathrm{Ca}(\langle[\mathrm{Ca} / \mathrm{Fe}]\rangle=$ $-0.06 \pm 0.06$ dex) in 47 Tuc. There is a much larger scatter in the abundance derived for $\mathrm{Na}(\langle[\mathrm{Na} / \mathrm{Fe}]\rangle=$ $-0.05 \pm 0.14 \mathrm{dex})$ and the $\mathrm{Na}$ abundance was found to correlate with $\mathrm{CN}$ strength. This is a phenomenon also previously observed in 47 Tuc stars (Cottrell \& Da Costa 1981; Cannon et al. 2003).

The analysis of the high-resolution spectrum of Lee 2525 determined an enhancement in the $\mathrm{Zr}$ abundance for this $\operatorname{star}[\mathrm{Zr} / \mathrm{Fe}]=+0.28$ dex relative to Arcturus. The medium-resolution spectra did not reflect this enhancement $([\mathrm{Zr} / \mathrm{Fe}]=+0.02$ dex relative to Arcturus). The mean $\mathrm{Zr}$ abundance (relative to Arcturus) for the survey subset showed an enhancement, $\langle[\mathrm{Zr} / \mathrm{Fe}]\rangle=+0.14 \pm$ 0.09 dex, but the large uncertainty is most likely due to the weakness and blending of the measured $\mathrm{Zr}$ feature. The mean $\mathrm{Ba}$ abundance is $\langle[\mathrm{Ba} / \mathrm{Fe}]\rangle=+0.28 \pm 0.05 \mathrm{dex}$, indicative of a homogeneous distribution of this element in 47 Tuc. The spectral features for $\mathrm{La}$ and $\mathrm{Nd}$ were deemed to be too blended at this resolution for reliable spectrum synthesis analysis, hence this resolution is too low to carry out a comprehensive survey of $s$-process elements in 47 Tuc giant stars.

Most of the light and heavy elements measured in this sample of stars, that all have very similar stellar parameters, have a small spread in values indicating that these elements were most likely created in nuclear processes prior to the formation of these stars. The relation of $\mathrm{Na}$ to $\mathrm{CN}$ gives the possibility of distinguishing between stellar populations within 47 Tuc. The $\mathrm{CN}$-weak population is typically considered as the initial population, for which the high-mass stars have evolved through stellar death and polluted the interstellar medium with products of $\mathrm{CNO}$ 
nucleosynthesis, including the $\mathrm{Na}$ enhancement that can be produced through the NeNa cycle. Hence the secondary population are the $\mathrm{CN}$-strong stars. This is consistent with the recent study by Milone et al. (2011), who used precision photometry from the Hubble Space Telescope and ground-based telescopes to identify multiple stellar populations in 47 Tuc. They note 2 major populations with $\mathrm{C}, \mathrm{N}$ and $\mathrm{Na}$ abundance characteristics similar to those found in our study.

The trend of $\mathrm{Na}$ and $\mathrm{CN}$ can be viewed as a continuum of $\mathrm{Na}$ to $\mathrm{CN}$ enhancement. From the baseline of enhancement within the initial $\mathrm{CN} / \mathrm{Na}-w e a k$ population the $\mathrm{CN}$-strong stars have been further enriched in $\mathrm{CN}$ and $\mathrm{Na}$ in relative proportions indicating the same process is responsible (CNO cycling via hot bottom burning). However this process was apparently activated in the $\mathrm{CN}$-strong population, not the $\mathrm{CN}$-weak population. So why do the $\mathrm{CN}$-strong stars have differing degrees of enhancement in $\mathrm{CN}$ and $\mathrm{Na}$ ? Is there another parameter that must be considered? Another consideration is that this sample resides at the connection of the AGB to the RGB. This may provide another distinction with which to understand the spread in $\mathrm{CN}$ and $\mathrm{Na}$ abundances.

The Lee 2525 sample results of the 47 Tuc giant stars provide an indication about the abundance patterns observed in the light elements. More detailed analysis of the whole sample of 47 Tuc giants will work with the best stellar atmosphere model for each star and to then determine stellar elemental abundances by spectrum synthesis. The heavy elements will require observations at a higher resolution to obtain better detail in the stellar spectra of these typically weak features.

\section{Acknowledgments}

CCW and PLC both wish to acknowledge the hospitality and financial assistance of the Max Planck Institute for Astrophysics which enabled progress to be made on this paper. PLC also wishes to acknowledge the support of the University of Canterbury for his sabbatical during 2011 and a Marsden Fund grant administered by the Royal Society of New Zealand.

This research has made use of the SIMBAD database, operated at CDS, Strasbourg, France and has made use of data products from the Two-Micron All-Sky Survey, which is a joint project of the University of Massachusetts and the Infrared Processing and Analysis Center, California Institute of Technology, funded by the National Aeronautics and Space Administration and the National Science Foundation.

\section{References}

Alonso, A., Arribas, S. \& Martínez-Roger, C., 1999, A\&AS, 140, 261

Briley, M. M., Harbeck, D., Smith, G. H. \& Grebel, E. K., 2004, AJ, 127,1588

Brown, J. A. \& Wallerstein, G., 1992, AJ, 104, 1818

Busso, M., Gallino, R., Lambert, D. L., Travaglio, C. \& Smith, V. V., 2001, ApJ, 557, 802

Campbell, S. W., Lattanzio, J. C., Elliott, L. M., 2006, ArXiv Astrophysics e-prints

Campbell, S. W., Yong, D., Wylie-de Boer, E. C., Stancliffe, R. J., Lattanzio, J. C., Angelou, G. C., Grundahl, F. \& Sneden, C., 2010, MemSaIt, 81, 1004

Cannon, R. D., Croke, B. F. W., Bell, R. A., Hesser, J. E. \& Stathakis, R. A., 1998, MNRAS, 298, 601

Cannon, R., da Costa G., Norris, J., Stanford, L., Croke, B., 2003, in New Horizons in Globular Cluster Astronomy Vol. 296 of the Astronomical Society of the Pacific Conference Series, Spectroscopy of Main Sequence Stars in Globular Clusters, ed. G. Piotto, G. Meylan, S. G. Djorgovski \& M. Riello, 175

Cottrell, P. L. \& da Costa, G. S., 1981, ApJL, 245, L79

Gratton, R., Sneden, C. \& Carretta, E., 2004, ARA\&A, 42, 385

Hinkle K. Wallace L., 2005, in Cosmic Abundances as Records of Stellar Evolution and Nucleosynthesis Vol. 336 of Astronomical Society of the Pacific Conference Series, The Spectrum of Arcturus from the Infrared through the Ultraviolet, ed. T. G. Barnes III \& F. N. Bash, 321

Karakas, A. I. \& Lugaro, M., 2010, PASA, 27, 227

Lee, S. W., 1977, A\&AS, 27, 381

Lind, K., Asplund, M., Barklem, P. S. \& Belyaev, A. K., 2011, A\&A, 528, A103+

Milone, A. P., et al., 2011, ArXiv e-prints, 1109.0900

Norris, J. \& Freeman, K. C., 1979, ApJL, 230, L179 (NF79)

Paltoglou, G. Freeman K. C. 1984, PhD Thesis, Mt. Stromlo and Siding Spring Observatories, Institute of Advanced Studies, Australian National University (PF84)

Reschenhofer, E., 2001, Journal of Statistics Education, 9

Sharp, R., et al., 2006, in Ground-based and Airborne Instrumentation for Astronomy Vol. 6269 of Proceedings of the SPIE, Performance of AAOmega: The AAT Multi-purpose Fiber-fed Spectrograph, I. S. McLean \& I. Masanori, 62690G

Skrutskie, M. F., et al., 2006, ApJ, 131, 1163

Sneden, C., 1973, PhD Thesis, University of Texas at Austin

Sneden, C., Ivans, I. I. \& Kraft, R. P., 2000, MemSaIt, 71, 657

Worley, C. C., Cottrell, P. L. \& Wylie de Boer, E. C., 2008, PASA, 25,53

Worley, C. C., Cottrell, P. L., Freeman, K. C. \& Wylie-de Boer, E. C., 2009, MNRAS, 400, 1039

Worley, C. C., Cottrell, P. L., McDonald, I. \& van Loon, J. T., 2010, MNRAS, 402, 2060

Wylie, E. C., Cottrell, P. L., Sneden, C. A. \& Lattanzio, J. C., 2006, ApJ, 649, 248 


\section{A Appendix 1}

Table A1. Photometry, effective temperature and surface gravity calculated from $V-K$, and $C N$ excess calculated in this study for the 97 giant stars in our survey of 47 Tuc

\begin{tabular}{|c|c|c|c|c|c|c|}
\hline Star ID & $V^{*}$ & $B-V$ & $V-K \#$ & $T_{\text {eff }}(V-K)$ & $\log g(V-K)$ & $\delta C_{2011}$ \\
\hline Lee4472 & 12.40 & 1.41 & 0.34 & 4274 & 1.44 & -0.112 \\
\hline Lee1704 & 13.00 & 1.25 & -0.89 & 4827 & 1.93 & -0.045 \\
\hline Pal502 & 12.64 & 1.36 & 3.28 & 4158 & 1.27 & -0.037 \\
\hline Lee1628 & 12.94 & 1.22 & 2.99 & 4339 & 1.50 & -0.017 \\
\hline Lee1316/Pal881 & 13.17 & 1.17 & 2.89 & 4411 & 1.64 & -0.014 \\
\hline Pal1151 & 13.00 & 1.25 & 3.06 & 4295 & 1.50 & -0.011 \\
\hline Lee2306/Pal388 & 12.59 & 1.29 & 3.07 & 4288 & 1.33 & -0.005 \\
\hline Lee 5601 & 12.88 & 1.25 & 3.06 & 4292 & 1.45 & -0.001 \\
\hline Lee2428 & 13.23 & 1.16 & 2.96 & 4360 & 1.63 & 0.012 \\
\hline Lee3622/Pal726 & 12.60 & 1.31 & 3.24 & 4183 & 1.27 & 0.014 \\
\hline Lee1506 & 13.27 & 1.15 & 2.87 & 4423 & 1.69 & 0.016 \\
\hline Lee1104/Pal436 & 13.78 & 1.08 & 1.72 & 4714 & 2.07 & 0.017 \\
\hline Lee1312/Pal771 & 14.48 & 1.05 & 2.57 & 4927 & 2.46 & 0.021 \\
\hline Lee1320/Pal925 & 13.52 & 1.13 & 1.57 & 4509 & 1.84 & 0.022 \\
\hline Lee3207/Pal380 & 13.11 & 1.22 & 2.97 & 4352 & 1.58 & 0.028 \\
\hline Lee2608 & 12.87 & 1.22 & 3.07 & 4285 & 1.44 & 0.031 \\
\hline Lee4737 & 12.96 & 1.25 & 3.10 & 4267 & 1.46 & 0.031 \\
\hline Lee4628 & 12.53 & 1.31 & 3.14 & 4241 & 1.28 & 0.043 \\
\hline Lee3305/Pal461 & 13.03 & 1.23 & 3.02 & 4319 & 1.53 & 0.044 \\
\hline Lee1522 & 13.56 & 1.08 & 1.88 & 4519 & 1.87 & 0.046 \\
\hline Lee1423/Pal1124 & 13.75 & 1.03 & 1.68 & 4761 & 2.08 & 0.047 \\
\hline Lee4506 & 13.54 & 1.11 & 1.73 & 4454 & 1.82 & 0.050 \\
\hline Pal1158 & 13.20 & 1.13 & 2.63 & 4617 & 1.78 & 0.052 \\
\hline Lee2616 & 13.21 & 1.14 & 2.84 & 4448 & 1.68 & 0.053 \\
\hline Lee2737 & 13.51 & 1.13 & 1.51 & 4553 & 1.87 & 0.053 \\
\hline Lee2742 & 13.04 & 1.08 & 2.89 & 4406 & 1.59 & 0.059 \\
\hline Lee3404/Pal440 & 14.53 & 0.90 & 2.57 & 4961 & 2.49 & 0.061 \\
\hline Lee2311/Pal500 & 13.98 & 0.77 & 2.02 & 5708 & 2.58 & 0.065 \\
\hline Lee4502 & 13.28 & 0.96 & -0.51 & 4468 & 1.69 & 0.074 \\
\hline Pal256/Tuc14 & 14.00 & 0.85 & 2.06 & 5325 & 2.44 & 0.075 \\
\hline Lee2310/Pal423 & 14.50 & 0.94 & 2.46 & 4838 & 2.42 & 0.075 \\
\hline Lee1201/Pal507 & 12.88 & 1.26 & 3.10 & 4266 & 1.43 & 0.076 \\
\hline Lee1206/Pal638 & 14.13 & 0.84 & 3.37 & 5252 & 2.46 & 0.076 \\
\hline Lee 5705 & 13.86 & 1.11 & 2.42 & 4505 & 1.98 & 0.077 \\
\hline Lee3306/Pal447 & 14.04 & 0.90 & 2.03 & 5288 & 2.44 & 0.079 \\
\hline Lee 2525 & 12.43 & 1.29 & 3.16 & 4232 & 1.23 & 0.081 \\
\hline Chu4432/W66 & 12.55 & 1.34 & 3.24 & 4181 & 1.25 & 0.083 \\
\hline Lee1313 & 14.44 & 1.00 & 3.08 & 4855 & 2.40 & 0.084 \\
\hline Lee1105 & 13.69 & 1.10 & 2.46 & 4598 & 1.97 & 0.086 \\
\hline Lee4514 & 14.35 & 0.98 & 2.34 & 4778 & 2.33 & 0.087 \\
\hline Lee1315/Pal869 & 14.10 & 0.81 & 2.85 & 5297 & 2.47 & 0.090 \\
\hline Lee3307 & 14.23 & 0.96 & 2.54 & 5448 & 2.58 & 0.092 \\
\hline Lee3201 & 13.78 & 0.88 & 1.81 & 5212 & 2.31 & 0.093 \\
\hline Lee1529 & 13.90 & 0.86 & 2.80 & 5118 & 2.31 & 0.096 \\
\hline Lee 8528 & 13.72 & 0.92 & 2.22 & 5031 & 2.20 & 0.105 \\
\hline Lee8302/Pal1446 & 14.06 & 0.85 & 1.78 & 5334 & 2.47 & 0.106 \\
\hline Pal306/Tuc32 & 14.02 & 0.85 & 2.03 & 5276 & 2.43 & 0.107 \\
\hline Lee1516 & 13.94 & 1.04 & 2.22 & 4660 & 2.10 & 0.110 \\
\hline Lee3312/Pal466 & 14.11 & 0.88 & 1.88 & 5303 & 2.48 & 0.115 \\
\hline Lee1207/Pal5628 & 14.07 & 0.84 & 2.06 & 5262 & 2.44 & 0.127 \\
\hline Lee1735 & 13.35 & 1.09 & 2.76 & 4505 & 1.77 & 0.128 \\
\hline Pal452 & 12.94 & 1.24 & 2.93 & 4378 & 1.53 & 0.131 \\
\hline Lee 5703 & 12.53 & 1.28 & 3.17 & 4221 & 1.26 & 0.131 \\
\hline Pal1036 & 13.95 & 0.86 & 2.08 & 5341 & 2.43 & 0.131 \\
\hline Lee2739 & 13.22 & 1.18 & 2.80 & 4472 & 1.70 & 0.132 \\
\hline Lee1513 & 12.41 & 1.32 & 3.14 & 4242 & 1.23 & 0.135 \\
\hline Pal487 & 13.17 & 1.15 & 2.87 & 4422 & 1.65 & 0.139 \\
\hline Lee3205/Pal351 & 14.07 & 0.83 & 2.05 & 5307 & 2.46 & 0.139 \\
\hline Lee2601 & 13.83 & 1.06 & 3.12 & 4638 & 2.04 & 0.144 \\
\hline
\end{tabular}


Table A1. (Continued)

\begin{tabular}{|c|c|c|c|c|c|c|}
\hline Star ID & $V^{*}$ & $B-V$ & $V-K \#$ & $T_{\text {eff }}(V-K)$ & $\log g(V-K)$ & $\delta \mathrm{C}_{2011}$ \\
\hline Chu4684W139 & 12.70 & 1.12 & 3.17 & 4223 & 1.33 & 0.146 \\
\hline Lee8202/Pal1526 & 14.08 & 0.86 & 3.04 & 5341 & 2.48 & 0.148 \\
\hline Lee3415/Pal368b & 14.03 & 0.86 & 1.99 & 5310 & 2.45 & 0.149 \\
\hline Lee3403/Pal428 & 14.03 & 0.84 & 2.79 & 5383 & 2.48 & 0.149 \\
\hline Lee2108/Pal342 & 14.01 & 0.84 & 2.47 & 5347 & 2.45 & 0.157 \\
\hline Lee3310/Pal474 & 14.07 & 0.85 & 2.92 & 5316 & 2.47 & 0.158 \\
\hline Lee1301/Pal516 & 12.64 & 1.22 & 2.91 & 4391 & 1.42 & 0.159 \\
\hline Lee2604 & 13.07 & 1.04 & 2.52 & 4731 & 1.79 & 0.161 \\
\hline Lee 8508 & 13.80 & 0.87 & 2.19 & 5006 & 2.22 & 0.162 \\
\hline Pal578 & 13.38 & 1.14 & 2.78 & 4488 & 1.77 & 0.164 \\
\hline Pal262/Tuc16 & 12.72 & 1.29 & 3.14 & 4240 & 1.35 & 0.166 \\
\hline Lee4602 & 13.07 & 1.19 & 2.99 & 4338 & 1.55 & 0.167 \\
\hline Lee2201/Pal368a & 13.97 & 0.83 & 1.90 & 5351 & 2.44 & 0.170 \\
\hline Chu4241/W164 & 12.62 & 1.12 & 3.05 & 4300 & 1.35 & 0.173 \\
\hline Lee3519 & 14.40 & 1.02 & 2.38 & 4755 & 2.34 & 0.174 \\
\hline Lee8301/Pal1353 & 13.96 & 0.85 & 2.33 & 5334 & 2.43 & 0.175 \\
\hline Pal571 & 13.75 & 1.08 & 1.63 & 4619 & 2.00 & 0.175 \\
\hline Lee1319 & 13.08 & 0.93 & 2.28 & 5037 & 1.95 & 0.177 \\
\hline Lee1519 & 13.89 & 0.88 & 3.08 & 5299 & 2.39 & 0.181 \\
\hline Lee1304/Pal506 & 14.51 & 0.96 & 3.38 & 4794 & 2.40 & 0.185 \\
\hline Lee3206/Pal383 & 14.06 & 0.84 & 1.86 & 5303 & 2.46 & 0.186 \\
\hline Lee1309 & 13.20 & 1.04 & 2.61 & 4635 & 1.79 & 0.187 \\
\hline Lee2419/Pal478 & 14.48 & 0.96 & 2.56 & 4852 & 2.42 & 0.194 \\
\hline Lee1324/Pal1125 & 14.04 & 0.85 & 2.13 & 5287 & 2.44 & 0.194 \\
\hline Lee 4626 & 13.27 & 1.17 & 2.87 & 4422 & 1.69 & 0.196 \\
\hline Lee4509 & 12.99 & 1.21 & 3.01 & 4326 & 1.51 & 0.198 \\
\hline Lee1408/Pal598 & 13.49 & 1.09 & 2.69 & 4566 & 1.87 & 0.201 \\
\hline Lee2511 & 13.84 & 0.84 & 1.94 & 5202 & 2.33 & 0.201 \\
\hline Pal661 & 12.54 & 1.26 & 3.15 & 4233 & 1.28 & 0.202 \\
\hline Lee1419 & 14.19 & 0.97 & 3.37 & 4795 & 2.27 & 0.208 \\
\hline Lee 5717 & 13.16 & 1.16 & 2.94 & 4372 & 1.61 & 0.209 \\
\hline Lee 5514 & 14.15 & 1.00 & 2.21 & 4705 & 2.21 & 0.216 \\
\hline Lee1747/S364 & 12.45 & 1.26 & 3.07 & 4284 & 1.27 & 0.223 \\
\hline Lee4636 & 13.30 & 1.17 & 2.78 & 4493 & 1.75 & 0.233 \\
\hline Lee 3510 & 13.63 & 1.09 & 2.82 & 4780 & 2.04 & 0.235 \\
\hline Lee4515 & 14.49 & 0.98 & 2.45 & 4848 & 2.42 & 0.262 \\
\hline Lee2302/Pal397 & 14.07 & 1.07 & 2.12 & 4720 & 2.19 & 0.264 \\
\hline Lee2528/Pal625 & 13.54 & 1.12 & 1.52 & 4532 & 1.87 & 0.307 \\
\hline
\end{tabular}

$* B \& V$ from SIMBAD

\# $K$ from 2MAss 